\title{
Immunologie et recombinaison homologue : étude de l'ontogenèse des lymphocytes $T$
}

La recombinaison homologue permet, par mutagenèse insertionnelle, de supprimer la synthèse de molécules clés de la différenciation et de la fonction des cellules du système immunitaire, permettant ainsi de préciser le rôle de ces protéines. C'est ainsi qu'ont d'ores et déjà été obtenues des souris déficientes en cellules $T$ cytotoxiques (négatives pour la $\beta_{2}$ microglobuline ou le CD8), en cellules $\mathbf{T}$ auxiliaires (négatives pour les molécules de classe II du CMH ou le CD4), en interleukine 2 et en chấnes lourdes des immunoglobulines.

\section{Stéphane Viville}

\section{ADRESSE ET TIRÉS À PART}

S. Viville : docteur en pharmacie, stagiaire doctoral, boursier de la fondation Mérieux. Laboratoire de génćtique moléculaire des eucaryotes du Cnrs, Inserm U.184, biologie moléculaire ct génćtique. Institut de chimic biologique, faculté de médecinc, 11, ruc

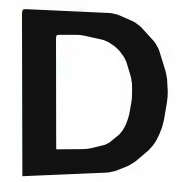

epuis le dix-neuvième siècle, un des principaux modes d'étude des systèmes vivants consiste à rechercher ou à créer des "monstres", cela afin de pouvoir comparer le normal et l'anormal, de comprendre l'importance et le rôle de tel ou tel organe. En deux siècles de recherche, les méthodes d'étude n'ont guère changé, seuls les instruments ont énormément évolué, permettant une analyse de plus en plus précise des phénomènes du vivant. On est ainsi passé de l'étude d'organes entiers à l'étude moléculaire de leur fonctionnement, de l'excision pure et simple d'organes à la création de souris, dont le génome porte la ou les mutations désirées [1].

En effet, l'utilisation de la recombinaison homologue $(\mathrm{RH})$ dans des cellules embryonnaires (ES) permet de créer des mutations préalablement choisies chez la souris [2, 3]. Actuellement, cette technique est surtout employée pour l'étude du développement embryonnaire et la compréhension du système immunitaire, mais elle peut aussi être utilisée plus généralement pour créer des modèles animaux de maladies.

Nous aimerions présenter dans cet article les travaux concernant l'utilisation de la recombinaison homologue en immunologie et plus précisément dans le développement des lymphocytes $T$.

La première caractéristique du système immunitaire est de pouvoir distinguer le soi du non-soi et, ainsi, de protéger l'organisme de toute invasion par un corps étranger, qu'il soit viral, bactérien ou parasitaire. Pour ce faire, l'ensemble des lymphocytes $\mathrm{B}$ ou $\mathrm{T}$ intervenant dans la réponse immunitaire vont subir, au cours de leur différenciation, deux 
sortes de sélection, l'unc permettant l'élimination des cellulcs potentiellement autoréactives et l'autrc, la sauvegarde des ccllules fonctionnclles $[4,5]$.

La " tolérance " immunitairc, terme générique englobant un grand nombre de processus, repose sur ces mécanismes de sélection. L'élimination des lymphocytes $T$ potentiellement autoréactifs ainsi que la restriction aux molécules du complexe majcur d'histocompatibilité ( $\mathrm{CMH})$ sc fait principalement dans le thymus [6]. En cffet, c'est dans le thymus que les ccllules pré-T vont réarranger les gènes codant pour les récepteurs des ccllules $T$. Les lymphocytes $\mathrm{T}$ dont le réccptcur (TcR) est susceptible de reconnaître un antigènc exogène présenté par des moléculcs de classe I ou de classe II du complexe majcur d'histocompatibilité $(\mathrm{CMH})$, vont être sauvegardés et sc différencicr en cellules $\mathrm{T}$ cytotoxiques ou auxiliaires respectivement, on parle de sélection positive [7, 8], cclle-ci prendrait place dans le cortex thymique [9]. En revanche, les lymphocytes $\mathrm{T}$ autoréactifs $[10,11]$, ou présentant une fortc affinité pour les molécules du $\mathrm{CMH}$, sont éliminés, on parle de sélection négative ou d'établissement de la tolérance au soi (figure 1).

Cette différenciation peut être suivie par l'apparition ou la disparition de certains co-réccptcurs à la surface des cellules T. Ainsi les cellules pré-T sont $\mathrm{TcR}^{-}, \mathrm{CD}^{-}{ }^{-}, \mathrm{CD}^{-}{ }^{-}, \mathrm{CD} 8^{-}$ (ces cellules sont dites " double négatives " car n'exprimant ni CD4, ni CD8) puis vont faiblement exprimer lcurs TcR et CD3, CD4 et CD8, on parle de cellules "doubles positives" (DP), avant d'être sélectionnées et de quitter le thymus sous forme de cellules "simples positives" (SP) c'està-dire $\mathrm{TcR}{ }^{+}, \mathrm{CD}^{+}$et $\mathrm{CD} 4{ }^{+} \mathrm{CD} 8$ ou CD4-CD8 + fonctionnelles.

Les lymphocytes $\mathrm{T}$ auxiliaires correspondent aux cellules SP $\mathrm{CD} 4{ }^{+} \mathrm{CD} 8-$, leurs réceptcurs sont susceptibles de reconnaître des peptides antigéniques présentés par des molécules de classe II du $\mathrm{CMH}$; on dit que ces lymphocytes sont restreints aux molécules de classe II. En revanche, les lymphocytes $\mathrm{T}$ cytotoxiques se retrouvent dans la périphérie sous forme $\mathrm{SP} \mathrm{CD}^{-}{ }^{-\mathrm{CD}} 8^{+}$et sont $\mathrm{m} / \mathrm{s} n^{\circ} 1$, vol. \&, janvier 92

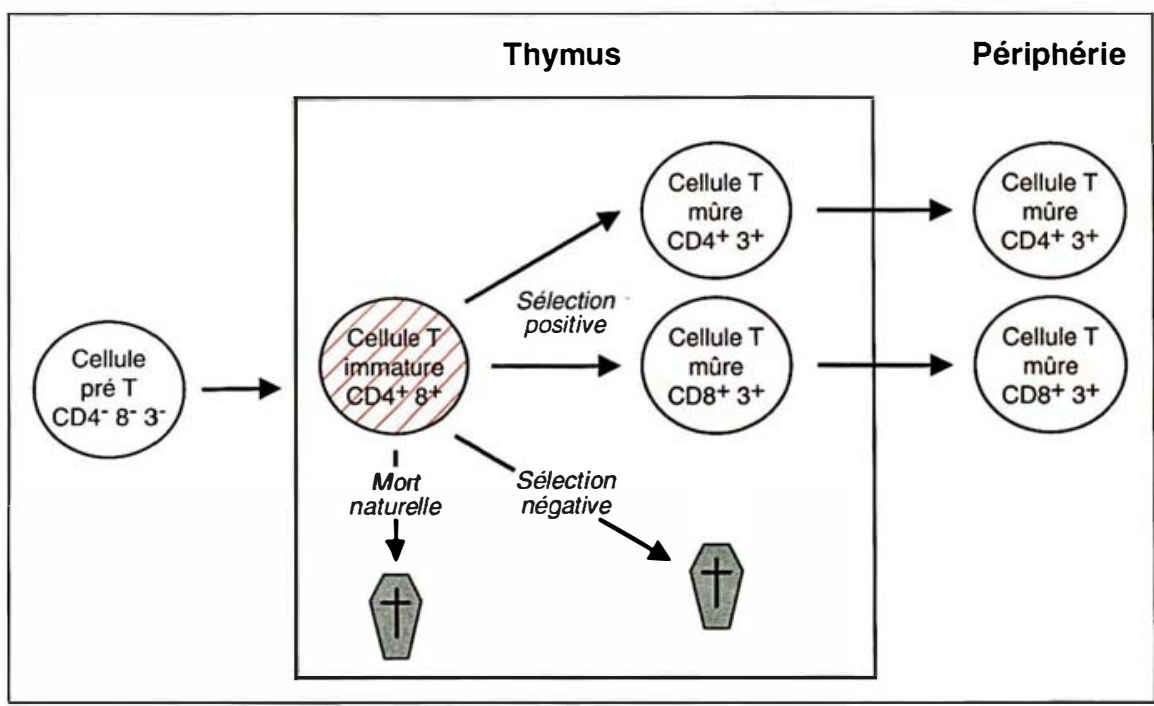

Figure 1. Ontogenèse des cellules $T$. Les cellules pré-T provenant de la moelle osseuse colonisent le thymus, où elles vont être sélectionnées et se différencier. Cette sélection permet de sauvegarder les cellules $T$ fonctionnelles (sélection positive) et d'éliminer les cellules autoréactives (sélection négative) : avant de rejoindre la périphérie sous forme de cellules mûres.

restrcints aux molécules de classe I (figure 2, p. 36). C'est afin de micux comprendre l'ensemble des réactions régissant la réponse immunitairc et, plus particulièrement, l'établissement de la tolérance, que différents laboratoires ont créé, par recombinaison homologue, des souris mutantes n'exprimant plus certains des gènes impliqués dans de tels processus.

Nous allons décrire les travaux concernant les souris déficientes en moléculcs de classe I ou II du $\mathrm{CMH}$ ainsi que les souris mutantes pour les coréceptcurs CD4 ou CD8.

\section{La recombinaison homologue et les cellules embryonnaires}

La recombinaison homologue est un phénomène d'échange entre deux molécules d'ADN présentant des homologies de séquence. Cela permet, par la construction de vectcurs appropriés, de muter un gène au sein du génome [1].

L'utilisation de cettc technique sur des cellules embryonnaires (ES) permet de créer des souris transgéniques portant une mutation préalablement choisic. En cffet, les ccllules ES ont la capacité, après injection dans un blastocystc, de coloniser la masse cellulaire interne et de participer au dévcloppement de l'embryon donnant naissance à une souris ditc "chimère " ou " mosaïque " puisque formée de ccllules injectées et de cellules du blastocyste [2]. Après croisement des souris chimères, on peut obtenir des souris dont le patrimoinc génétique provient des cellules injectécs et qui sont donc mutantes [3]. Chez la souris, la plupart des mutations étant récessives, l'ćtude des mutants se fait sur les souris homozygotes obtcnues après des croiscments successifs.

\section{Mutation de la $\beta_{2}$ microglobuline}

Les molécules de classe I, qui sont présentes à la surface de presque toutcs les ccllules, sont responsables de la présentation de l'antigène (généralement d'origine endogène, à la suite, par exemple, d'unc infection virale) aux lymphocytes $\mathrm{T}$ cytotoxiques, caractérisés par le co-récepteur CD8. Il existe chez un individu un grand nombre de molécules de classe I, mais ces molécules ne peuvent être 


\section{RÉFÉRENCES}

1. Capecchi MR. Altering the genome by homologous recombination. Science 1989 ; 244 : 1288-92.

2. Robertson EJ. Pluripotential stem cell lincs as a routc into the mouse germ line. Trends in Genetics 1986; 1 : 9-13.

3. Lemarchandel V, Montagutelli X. La recombinaison homologue: de nouvclles perspectives pour la transgenèse chez les mammifc̀res. médecine-sciences $1990 ; 6$ : 18-29.

4. Von Bochmer H, Teh HS, Kisiclow P. The thymus selects the useful, neglects the uscless and destroys the harmful. Immunol Today 1989 ; $10: 57-61$.

5. Ramsdell F, Fowlkes BJ. Clonal deletion versus clonal ancrgy : the role of the thymus in inducing sclf tolcrance. Science 1990 ; $248: 1342-8$.

6. Nikolic-Zugic J. Phenotypic and functional stages in the intrathymic development of $\alpha \beta$ T cclls. Immunol Today 1991; $2: 12$.

7. Marrack P, Kappler J. The T-cell repertoire for antigen and MHC. Immunol Today $1988 ; 9$ : 308-14.

8. Teh HS, Kisiclow $\mathrm{P}$, Scott $\mathrm{B}$, et al. Thymic major histocompatibility complex antigens and the $\alpha \beta$ T-cell receptor determine the CD4/CD8 phenotype of $\mathrm{T}$ cells. Nature 1988 ; 335 : 229-33.

9. Benoist C, Mathis D. Positive selection of the $T$ cell repertoire : where and when does it occur? Cell 1989 ; 58 : 1027-33.

10. Kappler JW, Roehm N, Marrack P. T cell tolcrance by clonal climination in the thymus. Cell $1987 ; 49: 237-80$.

11. Kisiclow $\mathrm{P}$, Blüthmann $\mathrm{H}$, Staerz UD, Stcinmetz $\mathrm{M}$, von Bochmer $\mathrm{H}$. Tolerance in $\mathrm{T}$-ccll-receptor transgenic mice involves deletion of nonmaturc $\mathrm{CD} 4{ }^{+} \mathrm{CD} 8{ }^{+}$thymocytes. Nature 1988 ; 333 : 742-6.

12. Zijlstra M, Li E, Saijadi F, Subramani $\mathrm{S}$, Jcanisch R. Germ-linc transmission of a disrupted $\beta 2$-microglobulin gene produced by homologous recombination in cmbryonic stem cclls. Nature $1989 ; 342$ : 435-8.

13. Koller $\mathrm{BH}$, Smithies O. Inactivating the $\beta 2$-microglobulin locus in mousc cmbryonic stem cclls by homologous recombination. Proc Natl Acad Sci USA 1989 ; 86 : 8932-5.

14. Zijlstra M, Bix M, Simister NE, Loring JM, Raulet DH, Jacnisch R. $\beta 2$-microglobulin deficicnt mice lack $\mathrm{CD}^{-}{ }^{-} \mathrm{CD} 8{ }^{+}$cytolytic T cells. Nature 1990 ; 344 : 742-6

15. Koller BH, Marrack P, Kappler JW, Smithies O. Normal development of mice deficient in $\beta 2 \mathrm{M}, \mathrm{MHC}$ class I protcins, and $\mathrm{CD}^{+}{ }^{+} \mathrm{T}$ cclls. Science $1990 ; 248$ :

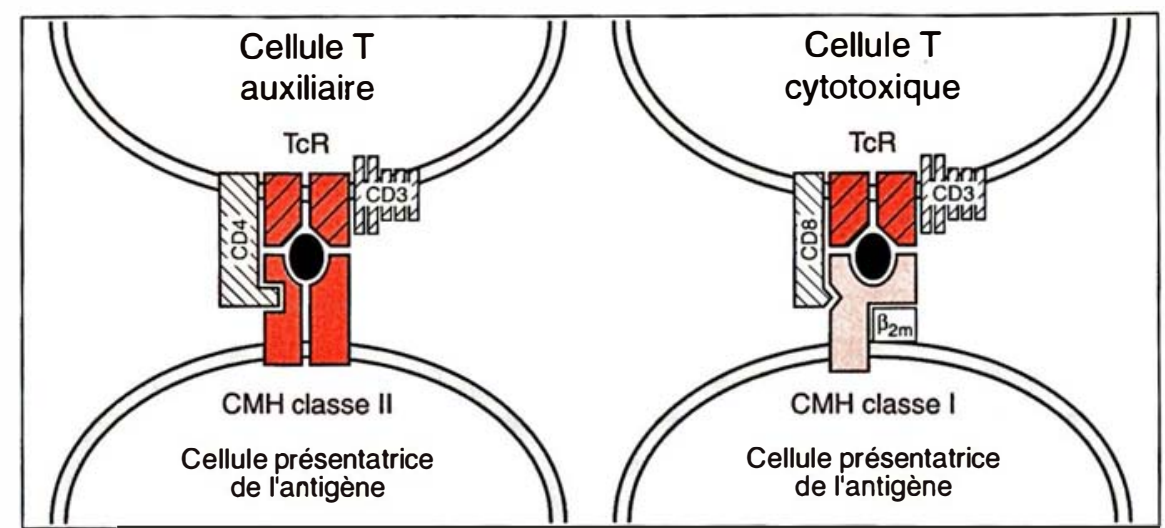

Figure 2. Présentation de I'antigène aux récepteurs $T$. Les peptides antigéniques sont présentés aux cellules $T$ auxiliaires $\left(C D 4^{+}, C D 3^{+}\right)$par les molécules de classe II du MHC. Ces cellules sont dites restreintes aux molécules de classe II. En revanche, les cellules $T$ cytotoxiques $\left(C 8^{+}, C D 3^{+}\right)$reconnaissent l'antigène quand il est associé aux molécules de classe I du MHC. Ces cellules sont restreintes aux molécules de classe I.

exprimées à la surface des cellules qu'en présence d'une molécule annexe, la $\beta_{2}$-microglobuline $\left(\beta_{2} \mathrm{~m}\right)$. Les groupes de $\mathrm{R}$. Jaenisch et de $\mathrm{O}$. Smithies ont ainsi pu créer des souris n'exprimant pas de molécules de classe I à la surface de leurs cellules en mutant par recombinaison homologue le gène de la $\beta_{2} \mathrm{~m}[12,13]$. Ces souris ne produisent pas de $\beta_{2} \mathrm{~m}$ et, comme prévu, elles n'expriment pas les molécules de classe I du $\mathrm{CMH}$ à la surface des cellules. Contrairement aux prévisions, cela n'a pas de retentissement majeur sur leur état général ; ces souris se développent et se reproduisent normalement.

L'analyse plus fine [14-16] de ces souris a permis :

(1) de confirmer que la $\beta_{2} m$ est indispensable à l'expression des molécules de classe I (au moins sous une forme fonctionnelle) à la surface des cellules ;

(2) de démontrer le rôle primordial des molécules de classe I dans l'ontogenèse des lymphocytes $\mathrm{T}$ cytotoxiques, résultat confirmant ceux obtenus par l'injection d'anticorps monoclonaux anti-classe I à des nouveaunés de souris [17]. En effet, ces souris sont dépourvues de lymphocytes $\mathrm{T}$ cytotoxiques mûres (T CD4CD8 $\left.{ }^{+}\right)$. L'analyse du thymus montre que les lymphocytes auxiliaires ( $\mathrm{T}$ $\mathrm{CD}^{+}{ }^{+} \mathrm{CD}^{-}$) et les précurseurs des deux types de cellules $\mathrm{T}$ (cellules DP) ne sont pas affectés par cette mutation. Il est à noter que si la proportion de cellules $\mathrm{T} \mathrm{CD4}^{+}$dans le thymus est identique chez la souris mutante et chez la souris normale, dans les organes lymphoïdes périphériques (rate ou ganglions lymphatiques), cette population est augmentée d'une proportion correspondant à celle des cellules $\mathrm{T} \mathrm{CD}^{+}$ manquantes.

(3) de démontrer que la maturation des lymphocytes $\mathrm{T}$ exprimant les récepteurs $\gamma \delta$ est indépendante des molécules de classe I. Ce résultat vient contredire des observations précédentes, qui laissaient supposer une sélection de ce type de lymphocytes $\mathrm{T}$ par les molécules de classe I ;

(4) de démontrer que, contrairement à certaines hypothèses, la $\beta_{2} \mathrm{~m}$ n'a pas de fonction chimio-attractive sur les lymphocytes immatures et par conséquent pas sur le développement du thymus.

Paradoxalement, les greffes de cellules hématopoiétiques de souris mutantes pour la $\beta_{2} \mathrm{~m}$ (donc n'exprimant pas les molécules de classe I du $\mathrm{CMH}$ ) à des souris syngéniques irradiées sont rejetées. Ce phénomène, préalablement observé, est à imputer aux cellules spléniques NK (natural killer) comme le démontre l'étude de M. Bix et al. [18] qui arrive à inhiber le rejet en traitant les souris gref- 
fées par un anticorps anti-NK1.1. L'absence de molécules de classe I provoquerait la non-reconnaissance de ces cellules, comme appartenant au soi, par les cellules NK qui, de ce fait, les détruisent.

Nous attendons toujours les résultats concernant le comportement de ces souris déficientes en classe I lors d'une infection virale. Développeront-elles une réponse cytotoxique, le peu de cellules CD8 restantes (2\%) est-il suffisant pour établir une réponse cytotoxique ? La réponse humorale devrait être normale puisque les cellules $T$ auxiliaires ne sont pas affectées par la mutation.

Avant de conclure que les molécules de classe I n'ont aucun rôle dans l'embryogenèse, il serait bon de contrôler l'absence d'expression de formes de ces molécules à la surface cellulaire susceptibles d'intervenir au cours du développement.

\section{Création de souris mutantes pour CD8}

Chez la souris, la protéine CD8 est constituée de deux chaînes peptidiques, codées par les gènes $C D 8 \alpha$ et $C D 8 \beta$, liées entre elles par un pont disulfure.

CD8 $\alpha$ peut s'exprimer (sous forme d'homodimère) à la surface des cellules T, ce qui n'est pas le cas de CD8 $\beta$. C'est pour cette raison que W.P. Fung-Leung et al. [19] ont décidé de créer des souris mutantes pour la chaîne $\operatorname{CD} 8 \alpha$, afin de pouvoir étudier le rôle de CD8 dans la réponse immunitaire. Les souris homozygotes pour cette mutation sont saines et fertiles, leurs organes lymphoïdes sont anatomiquement normaux et ne présentent aucun signe d'atrophie. Dans les organes lymphoïdes périphériques (rate, ganglions lymphatiques), le rapport cellules $\mathrm{T}$ sur cellules $\mathrm{B}$ est normal. L'analyse par cytométrie de flux révèle l'absence de cellules T CD8 ${ }^{+}$, confirmant ainsi que la protéine CD8 $\beta$ ne peut pas s'exprimer seule à la surface des cellules $T$. Les premiers résultats suggèrent que, comme pour la mutation de la $\beta_{2} \mathrm{~m}$, l'absence de cellules $\mathrm{T} \mathrm{CD}^{+}{ }^{+}$est compensée dans les organes lymphoïdes périphériques par des cellules $\mathrm{T} \mathrm{CD}^{+}{ }^{+}$. Ces souris, comme on pouvait s'y attendre, ne développent pas de réponse cytotoxi- que, ni contre des cellules allogéniques ni contre des cellules infectées par le virus de la vaccine. Ces résultats prouvent la nécessité de la molécule CD8 dans l'ontogenèse des lymphocytes $\mathrm{T}$ cytotoxiques fonctionnels.

En revanche, cette mutation n'affecte pas l'ontogenèse des cellules $\mathrm{T}$ auxiliaires restreintes aux molécules de classe II du CMH. En effet, les lymphocytes $\mathrm{T} \mathrm{CD4}^{+}$isolés de rates de souris mutantes sont capables de proliférer et de sécréter de l'interleukine 2 (IL2) en réponse à une stimulation par des allo-antigènes. De plus, ces souris développent une réponse humorale normale après une vaccination avec un antigène dépendant des cellules $\mathrm{T}$ auxiliaires. Ainsi la maturation des cellules $\mathrm{T} \mathrm{CD}^{+}{ }^{+}$serait indépendante de l'expression du marqueur CD8. Ces résultats viennent confirmer et étendre ceux préalablement obtenus après in jection d'anticorps anti-CD8 à des souriceaux nouveau-nés [20,21], travaux qui n'avaient pas conduit à des conclusions sur le rôle fonctionnel de la protéine CD8.

Les souris $\beta_{2} \mathrm{~m}^{-}$et $\mathrm{CD} 8^{-}$seront très utiles dans l'étude de l'ontogenèse des lymphocytes $T$, des processus de réponse aux infections, des maladies auto-immunes et des rejets de greffes. De plus, la réintroduction par transgenèse de gène codant pour des molécules CD8 modifiées sur ces souris CD8- devrait permettre d'analyser la molécule CD8 et d'élucider le(s) mécanisme(s) de transduction des signaux qu'elle assure lors des présentations antigéniques.

\section{Mutation \\ chez la souris du co-récepteur CD4}

Les lymphocytes $T$ auxiliaires expriment à leur surface le co-récepteur CD4. Cette molécule, d'après les résultats obtenus par injection d'anticorps anti-CD4 à des souriceaux nouveau-nés, semble étroitement impliquée dans la sélection des cellules $\mathrm{T}$ au niveau du thymus $[20,22$, 23]. De plus, l'activation des lymphocytes $\mathrm{T}$ auxiliaires peut être bloquée, in vitro, par un anticorps antiCD4, suggérant que CD4 comme CD8 joue un rôle dans la transduction des signaux d'activation, cela en col-

laboration avec le complexe $\mathrm{CD} 3 / \mathrm{TcR}$

Afin de pouvoir étudier le rôle de CD4, A. Rahemtulla et al. [24] ont créé par recombinaison homologue des souris déficientes en co-récepteur CD4. Là-encore, les souris homozygotes ne présentent pas d'anomalies majeures.

L'analyse des organes lymphoïdes montre que le nombre de cellules y est normal mais qu'aucune de ces cellules n'exprime la molécule CD4. Dans le thymus, le nombre de cellules $\mathrm{CD}^{+}{ }^{+}$est comparable à celui de type sauvage. En revanche, ce nombre est augmenté, dans une proportion correspondant aux cellules $\mathrm{T} \mathrm{CD}^{+}$ manquantes, dans la rate et les ganglions lymphatiques. On peut noter la présence, dans les ganglions lymphatiques, d'une petite population de cellules $\mathrm{TcR}+\mathrm{CD} 4-\mathrm{CD} 8-$. Cela prouverait que la molécule CD4 n'a pas de rôle primordial dans l'ontogenèse des lymphocytes $T$ cytotoxiques.

Il serait intéressant de voir si, aussi bien pour cette mutation que pour la mutation de CD8, la population double positive précurseur des lymphocytes $\mathrm{T}$ simples positifs est toujours présente avec simplement un corécepteur en moins. De plus, l'étude du répertoire des récepteurs des cellules $T$ présentes dans chacune des souris de ce type devrait confirmer le rôle des molécules CD4 et CD8 dans la sélection positive.

Cette étude prouve l'importance des lymphocytes CD4 + dans la réponse immunitaire humorale. En effet, ces souris sont incapables de produire des anticorps anti-érythrocytes de mouton.

En, revanche, ces souris sont capables, et à un niveau similaire à celui de souris normales, d'établir une réponse cytotoxique après une infection virale.

On peut aussi se demander si ces souris constituent un modèle animal pour l'infection au virus HIV, dans la mesure où elles sont dépourvues de lymphocytes $\mathrm{T}$ auxiliaires. Réagirontelles aux infections comme des malades infectés par le virus HIV?

\section{Création de souris classe II négatives}

Les molécules de classe II du $\mathrm{CMH}$ s'expriment, contrairement aux molé- 


\section{RÉFÉRENCES}

16. Kahn A. Recombinaison homologuc, un nouvel outil au service des immunologistes des souris déficientes en $\beta 2$-microglobuline. médecine-sciences $1990 ; 6$ : 592-3.

17. Marusic-Galesic S, Stephany DA, Longo DL, Kruisbeck AM. Development of $\mathrm{CD}^{-} \mathrm{CD}^{+}$cytotoxic $\mathrm{T}$ cells requires interactions with class I MHC determinants. Nature 1988 ; 333 : 180-3.

18. Bix M, Liao N, Zijlstra M, Loring J, Jacnisch R, Raulet D. Rejection of class I MHC-deficient hacmopoietic cells by irradiated MHC-matched mice. Nature 1991 ; 349 : 329-31.

19. Fung-Lcung WP, Schilham MW, Rahemtulla A, et al. CD8 is necded for development of cytotoxic T-cells but not for helper T-cells. Cell 1991; 65 : 443-9.

20. Ramsdell F, Fowlkes BJ. Engagement of CD4 and CD8 accessory molecules is required for $\mathrm{T}$-cell maturation. J Immunol $1989 ; 143: 1467-71$.

21. Zùniga-Pflücker JC, Jones LA, Longo $\mathrm{DL}$, Kruisbeck AL. CD8 is required during positive sclection of $\mathrm{CD}^{-} / \mathrm{CD}^{+} \mathrm{T}$-cells. $J$ Exp Med 1990 ; 171 : 427-37.

22. MacDonald HR, Hengartner $H$, Pedrazzini T. Intrathymic deletion of selfreactive cells prevented by neonatal antiCD4 antibody treatment. Nature $1988 ; 335$ : 174-6.

23. Fowlkes BJ, Schwartz RH, Pardoll DM. Delction of sclf-rcative thymocytes occurs at a $\mathrm{CD}^{+}{ }^{+} \mathrm{CD} 8{ }^{+}$precursor stage. Nature 1988 ; 334 : 620-3.

24. Rahemtulla A, Fung-Lcung WP, Schliham, et al. Normal development and function of $\mathrm{CD}^{+}{ }^{+}$cclls but markedly decreased helper cell activity in micc lacking CD4 Nature 1991 ; 353 : 180-4.

25. Cosgrove D, Gray D, Dicrich A, et al. Mice lacking MHC class II molecules. Cell $1991 ; 66$ : 1051-66

26. Kruisbeck AM, Mond JJ, Fowlkes BJ, Carmen JA, Bridges S, Longo DL. Absence of the $\mathrm{Lyt}^{-2^{-}}$, $\mathrm{L} 3 \mathrm{~T}_{4}^{+}$lincage of $\mathrm{T}$ cells in mice treated neonatally with anti-IA correlates with absence of intrathymic I-A-bcaring antigen-presenting cell function. $J$ Exp Med $1985 ; 161: 1029-47$.

27. Grusby MJ, Johnson RS, Papaioannou VE, Glincher LH. Depletion of CD4 + Tcells in major histocompatibility complex class II-deficient micc. Science 1991; 253 : 1417-20.

28. Mombacrts P, Clarke AR, Hooper ML, Tonegawa S. Creation of a large genomic deletion at the $\mathrm{T}$-cell antigen receptor $\beta$-subunit locus in mouse embryonic stem cells by gene targeting. Proc Natl Acad Sci USA 1991; 88 : 3084-7.

29. Schorlc $H$, Holtschke $T$, Hünig $T$, Schimpl A, Horak I. Development and function of $\mathrm{T}$ cells in mice rendered interlcukin-2 deficient by genc targeting. Nature 1991 ; 352 : 621-4.

30. Kitamura D, Rocs J, Kühn R, Rajewsky K. A B cell-deficient mousc by targeted disruption of the membranc cxon of the immunoglobulin $\mu$ chain genc. Nature cules de classe I, dans un nombre très limité de cellules appartenant au système immunitaire. A la surface des macrophages et des cellules dendritiques (cellules présentatrices des antigènes), elles ont pour fonction de présenter les peptides antigéniques aux lymphocytes $\mathrm{T}$ auxiliaires. A la surface des lymphocytes B, elles participent à l'interaction entre cellules $\mathrm{B}$ et $\mathrm{T}$. Au niveau du thymus, où on les retrouve à la surface de certaines cellules du stroma, elles interviennent dans la sélection positive et négative des thymocytes CD4 ${ }^{+}$. Ces molécules sont étudiées depuis longtemps, mais un grand nombre de questions sur leur mécanisme d'action reste encore sans réponse. Par recombinaison homologue, des souris classe II négatives ont été créées afin d'analyser les divers rôles des molécules de classe II du CMH.

Il existe, chez la souris, deux complexcs de molécules de classe II, I-A et I-E. Les lignées de souris d'haplotype H-2b (C57BI/6 ; SJL ; 129sv) n'expriment pas le complexe I-E du fait d'une délétion dans la région promotrice du gène codant pour la chaînc E $\alpha$. En utilisant les cellules ES D3 provenant de souris 129 sv, il suffit donc, pour créer une lignéc de souris classe II négatives, de muter soit la chaîne $\mathrm{A} \alpha$ soit $\mathrm{A} \beta$. D. Cosgrove et al. [25] ont choisi de muter la chaîne $A \beta$. Chez les souris homozygotes pour la mutation $\mathrm{A} \beta$ (souris $\mathrm{A} \beta \%$ ) aucune expression de molécules de classe II, quelle que soit la forme, n'a pu être mise en évidence, aussi bien par cytométrie de flux, que par immunoprécipitation ou par immunohistologie.

L'analyse des organes lymphoïdes périphériques montre une diminution massive des cellules $\mathrm{T} \mathrm{CD}^{+}$et une augmentation proportionnelle des cellules $\mathrm{T} \mathrm{CD}^{+}$. Les lymphocytes $\mathrm{T}$ $\mathrm{CD} 4{ }^{+}$restants, qui représentent 3 à $7 \%$ de la population normale, sont d'un volume supérieur et expriment trois fois moins de TcR. Ces paramètres, associés à la présence de certains marqueurs cellulaires (Thy 1, CD5, CD44), laissent supposer que ces lymphocytes sont activés.

Dans le thymus, là aussi, la population de thymocytes simples positif $\mathrm{CD}^{+}{ }^{+}$est réduite mais de façon moins marquée que dans la périphé- ric. Outre lcur petite taille, les différents marquages effectués sur ces cellules (CD8 $8^{\text {faitlsc }} \mathrm{TcR}^{-}, \mathrm{CD}^{-}, \mathrm{J} 11 \mathrm{~d}^{+}$) suggèrent que ces cellules sont immatures.

Les cellules double négatives CD4 CD8 - ne présentent pas de changement notable. En revanche, les thymocytes DP CD4 + CD8+ expriment CD4 à un niveau plus élevé, alors que la population SP $\mathrm{CD}^{+}$ est peu affectée par cette mutation, parfois légèrement augmentée, compensant peut-être le manque de ccllules simples positives CD4 ${ }^{+}$.

Ces résultats, partiellement obtenus par injection d'anticorps monoclonaux anti-classe II à des souris nouveau-nées [26], apportent une preuve définitive du rôle des molécules de classe II dans la sélection positive des lymphocytes $\mathrm{T}$ auxiliaires. Il reste à déterminer quel est le mécanismc qui permet l'apparition de ccllules $\mathrm{CD}^{+}$dans la périphéric et pourquoi elles semblent activées. S'agit-il d'une sélection positive due à des molécules analogues aux molécules de classe II, d'unc sélection par des molécules de classc I ou simplement d'une erreur dans la sélection négative laissant échapper quelques cellules?

Ces souris permettent aussi l'étude du rôle des lymphocytes auxiliaircs dans l'ontogenèse des lymphocytes $B$. L'analyse des ganglions lymphatiques et de la rate des souris mutantes montre que les follicules sont dépourvus de centre germinatif.

La production de plasmocytes $\mathrm{IgM}^{+}, \mathrm{IgG}^{+}$semble normale, ce qui permet de dire que l'absence des classe II, et donc de cellules $\mathrm{T}$ auxiliaires, ne perturbe pas ou peu la différenciation des cellules $B$.

La synthèse d'anticorps après vaccination par des antigènes $T$ indépendants est normale. En revanche, chez les souris mutantes, il n'y a pas d'anticorps produits après vaccination par des antigènes $T$ dépendants.

Il serait intéressant de tester l'aptitude de ces souris à répondre à des infections comme nous l'avons fait à propos de souris $\mathrm{CD}^{\circ}$; on peut se demander si ces animaux dépourvus de cellules $\mathrm{T} \mathrm{CD}^{+}$représentent un modèle animal adapté à l'étude des affections qui accompagnent lc SIDA ? 
Le croisement des souris $\mathrm{A} \beta^{\circ}$ avec des souris n'exprimant que le complexc I-E du CMH ou l'exprimant de façon restreinte à certains compartiments tissulaires devrait permettre d'élucider un ccrtain nombre de questions. Existc-t-il une différence fonctionnclle entrc les molécules I-A et I-E ct cela sc traduit-il par une différence au niveau du répertoire des cellules $\mathrm{T}$ ainsi sélectionnées? Il pourrait permettre de confirmer la localisation de la sélection positive des thymocytes, au moins des lymphocytes T CD4.

Les travaux de Grusby et al. [27] viennent confirmer les résultats précédemment obtenus par D. Gosgrove.

\section{Conclusion}

Ces travaux démontrent, de façon définitive, que la sélection positive des cellules $T$ cytotoxiques ou auxiliaires se fait par un mécanisme similairc, la reconnaissance des molécules du $\mathrm{CMH}$, mais impliquant des molécules différentes, classe I ou classe II, respectivement. Ils mettent surtout en évidence l'indépendance qui existe dans la maturation des deux types de cellules T. En effet, l'absence de CD4 ou de CD8 n'affecte pas la maturation des lymphocytes respectivement cytotoxiques ou auxiliaires. Il scmble que le stade où les cellules $T$ expriment les deux marqueurs (cellules DP) ne soit qu'une facilité dans la différenciation : plutôt que de faire deux types de cellules immatures simples positives (SP), il est plus facile de n'en faire qu'un exprimant les deux marqueurs CD4 ct CD8. En effet, chez les souris mutantes pour l'un ou l'autrc, le stade de différcnciation correspondant au stade DP existe probablement mais sous forme SP, comme semble le démontrer la présence de thymocytes CD4 ${ }^{+} \mathrm{CD} 3-$ chez les souris mutantes pour CD8 [19].

Cela demande à être vérifié par l'utilisation d'autres marqueurs caractéristiques de ce stade de différenciation. En revanche, ces molécules (CD4, CD8) sont indispensables à l'obtention de lymphocytes $\mathrm{T}$ fonctionnels. L'obtention de souris déficientes en récepteurs $\mathrm{T} \alpha \beta$ par l'injection des cellules ES portant une large délétion,

Tableau I

IMMUNOLOGIE ET RECOMBINAISON HOMOLOGUE : LES MUTATIONS DÉJȦ OBTENUES

\begin{tabular}{|c|c|c|c|}
\hline Gène muté & $\begin{array}{l}\text { Cellules ES } \\
\text { utilisées }\end{array}$ & Phénotype & Références \\
\hline $\begin{array}{c}\beta_{2} \\
\text { microglobuline }\end{array}$ & D3 & \multirow{2}{*}{$\begin{array}{c}\text { Absence } \\
\text { de molécules } \\
\text { de classe } 1 \\
\text { du CMH } \\
\text { à la surface } \\
\text { des cellules }\end{array}$} & {$[12,14,18]$} \\
\hline $\begin{array}{c}\beta_{2} \\
\text { microglobuline }\end{array}$ & E14 & & {$[13,15]$} \\
\hline CD8 & D3 & $\begin{array}{c}\text { Absence de cellules } \\
T \text { cytotoxiques }\end{array}$ & [19] \\
\hline CD4 & D3 & $\begin{array}{c}\text { Absence de cellules } \\
T \text { auxiliaires }\end{array}$ & [24] \\
\hline $\mathrm{A} \beta \mathrm{CMH}$ & D3 & $\begin{array}{c}\text { Absence } \\
\text { de molécules de } \\
\text { classe II du CMH }\end{array}$ & {$[25,27]$} \\
\hline TCR- $\beta$ & E14 & Pas de souris & [28] \\
\hline $\mathrm{IL}-2$ & E14 & $\begin{array}{l}\text { Pas d'effet majeur } \\
\text { sur l'ontogenèse } \\
\text { des lymphocytes T }\end{array}$ & [29] \\
\hline $\lg M$ & D3 & $\begin{array}{c}\text { Absence de cellules } \\
\text { B mûres }\end{array}$ & [30] \\
\hline
\end{tabular}

obtenue par recombinaison homologue, dans le gène codant pour la région constante de la chaîne $\beta$ du $\mathrm{TcR}$ récemment décrite [28] complétcra cette étude de la différenciation des ccllules $T$.

De façon surprenante, les souris déficientes en interlcukinc 2 [29] ne présentent pas d'altération majeure dans la différenciation des lymphocytes $\mathrm{T}$. Cependant, il est à noter que les souris homozygotes analysées dans ces expériences proviennent toutes de parents hétérozygotes (communication personnelle), or il est probable que l'IL-2 maternelle agisse sur le développement du fœtus.

Un complément intéressant dans l'étude du système immunitaire viendra de l'analyse des souris déficientes en ccllules B mûres, obtenucs par mutation de la chaînc $\mu$ des immuno- globulines [30]. L'ensemble des souris mutantes déjà créécs par recombinaison homologue, et de celles à venir, forme un moyen d'investigation très puissant. L'exploitation de ces modèles en est encore à ses débuts ct représente un champ de recherches passionnant (Tableau I). De plus, le croisement de ces souris mutantes entre elles ou avec des souris issues de la transgenèse devrait permettre de compléter cettc étude du système immunitaire

\section{Remerciements}

L'auteur souhaite remercier les Drs C. Benoist et D. Mathis pour la lecture critique qu'ils ont faite de ce manuscrit, ainsi que la fondation Mérieux pour son aide financière. 


\section{Summary}

Immunology and homologous recombination : study of $T$ cells ontogeny

One of the most powerful methods for studying any living organism is to identify a "monster " that can be compared with its "normal » counterpart. Recently, it has become possible to create mice carrying almost any chosen mutation using homologous recombination. This technique has been particularly useful for studying the immune system. Here we discuss four different mutant mice, focusing on their relevance to $\mathrm{T}$-cell development : (1) mice unable to express $\beta-2$ microglobulin, a protein required for cell surface expression of major histocompatibility complex (MHC) class I molecules ; (2) mice in which expression of CD8, a coreceptor on class I restricted $\mathrm{T}$ cells, has been abrogated ; (3) mice in which expression of CD4, a coreceptor on class II restricted $\mathrm{T}$ cells, has been abrogated ; (4) mice lacking cell surface expression of MHC class II heterodimers due to a drastic mutation in the $A \beta$ gene. 